\title{
First Measurements of SuperCDMS SNOLAB 100 mm Diameter Germanium Dark Matter Detectors with Interleaved Charge and Phonon Channels
}

\author{
H. Chagani ${ }^{*},{ }^{a}$ D. A. Bauer, ${ }^{b}$ D. Brandt, ${ }^{c}$ P. L. Brink ${ }_{,}^{c}$ R. Bunker, ${ }^{d}$ B. Cabrera, ${ }^{e}$ \\ M. Cherry, ${ }^{c}$ G. A. Codoreanu, ${ }^{a}$ E. Do Couto e Silva, ${ }^{c}$ P. Cushman, ${ }^{a}$ G. L. Godfrey, ${ }^{c}$ \\ J. Hall, ${ }^{b}$ S. Hansen, ${ }^{b}$ J. Hasi, ${ }^{c}$ M. Kelsey, ${ }^{c}$ A. Kennedy, ${ }^{a}$ C. J. Kenney, ${ }^{c}$ S. W. Leman, ${ }^{f}$ \\ V. Mandic, ${ }^{a}$ N. Mirabolfathi, ${ }^{g}$ S. Monin, ${ }^{a}$ D. Nagasawa, ${ }^{e}$ L. Novak, ${ }^{e}$ R. Partridge, ${ }^{c}$ \\ C. Phenicie, ${ }^{a}$ K. Page, ${ }^{h}$ M. Pyle, ${ }^{g}$ R. Radpour, ${ }^{a}$ W. Rau, ${ }^{h}$ R. Resch, ${ }^{c}$ B. Sadoulet,${ }^{g}$ \\ D. N. Seitz, ${ }^{g}$ B. Serfass, ${ }^{g}$ B. Shank, ${ }^{e}$ D. Strandberg, ${ }^{a}$ A. Tomada, ${ }^{c}$ A. N. Villano, ${ }^{a}$ \\ J. Yen, ${ }^{e}$ B. A. Young ${ }^{i}$ and J. Zhang ${ }^{a}$
}

${ }^{a}$ School of Physics \& Astronomy, University of Minnesota, Minneapolis, MN 55455, USA

${ }^{b}$ Fermi National Accelerator Laboratory, Batavia, IL 60510, USA

' SLAC National Accelerator Laboratory, Menlo Park, CA 94025, USA

${ }^{d}$ Department of Physics, Syracuse University, Syracuse, NY 13244, USA

${ }^{e}$ Department of Physics, Stanford University, Stanford, CA 94305, USA

${ }^{f}$ Department of Physics, Massachusetts Institute of Technology, Cambridge, MA 02139, USA

${ }^{g}$ Department of Physics, University of California, Berkeley, Berkeley, CA 94720, USA

${ }^{h}$ Department of Physics, Physics Engineering \& Astronomy, Queen's University, Kingston, ON K7L 3N6, Canada

${ }^{i}$ Department of Physics, Santa Clara University, Santa Clara, CA 95053, USA

E-mail: chagani@physics.umn.edu

The first phase of the Super Cryogenic Dark Matter Search (SuperCDMS) SNOLAB experiment proposed is a $110 \mathrm{~kg}$ array of germanium and silicon athermal phonon detectors. It is expected to reach a better sensitivity by an order of magnitude than that which has been achieved so far by the best experiments in the field. The technical challenges of constructing a payload of this size have led to the development of $1.4 \mathrm{~kg}$ germanium detectors (100 mm diameter, $33 \mathrm{~mm}$ thick), which are 2.3 times larger than those presently in use in the SuperCDMS experiment at Soudan. The first results from testing of a prototype detector with interleaved phonon and ionization channels are presented. The test results are promising for the use of these detectors in the next phase of SuperCDMS.

Technology and Instrumentation in Particle Physics 2014

2-6 June, 2014

Amsterdam, the Netherlands

\footnotetext{
* Speaker.
} 


\section{Introduction}

The Super Cryogenic Dark Matter Search (SuperCDMS) experiment employs germanium crystals to perform direct searches for dark matter in the form of Weakly Interacting Massive Particles (WIMPs) [1]. The first phase, SuperCDMS Soudan, consists of 15 cylindrical germanium crystals, each of diameter $76 \mathrm{~mm}$, height $25 \mathrm{~mm}$ and mass $\sim 600 \mathrm{~g}$, which have been commissioned at Soudan Underground Laboratory. The detectors are operated at cryogenic temperatures of $\sim 50 \mathrm{mK}$ and event-by-event discrimination between electron and nuclear recoils is achieved through simultaneous collection of ionization and phonon signals. The phonon signal is used to determine the interaction energy independent of recoil type and the ionization signal distinguishes between these recoils. The ratio of ionization-to-phonon recoil energy is the discrimination parameter termed ionization yield. The ionization yield is lower for neutron and WIMP interactions than for electron recoils from most forms of background radiation (e.g. gamma-rays), and thus a nuclear recoil band can be defined in the plane of yield versus recoil energy that represents the WIMP signal region [目, 解.

Based on a concept first demonstrated by Luke et al. [П], each SuperCDMS Soudan detector, referred to as the interleaved Z-sensitive Ionization and Phonon (iZIP) detector [5], features interleaved phonon and ionization sensors on both sides of the crystal. The result is an electric field at the surface of the detector between the biased ionization electrode and grounded phonon sensor. Events interacting within $\sim 10 \mu \mathrm{m}$ from each crystal face, termed surface events, will only generate an ionization signal in the nearest face's instrumented ionization readout. This allows for rejection of these events [6], which were found to leak into the nuclear-recoil band due to incomplete charge collection and were a significant background in the CDMS-II experiment [2, 3].

The next phase of the SuperCDMS experiment will be a $110 \mathrm{~kg}$ total mass semiconductor detector array commissioned at the SNOLAB Underground Laboratory. The projected sensitivity of this larger array is at least $8 \times 10^{-47} \mathrm{~cm}^{2}$ for a WIMP mass of $50 \mathrm{GeV} / \mathrm{c}^{2}$. The increased mass of the SuperCDMS SNOLAB experiment [ [] poses significant challenges in the form of increased labor, cold hardware, warm electronics and heat load. The development of crystals larger than the present generation in use at the Soudan Underground Laboratory can alleviate these problems. In an initial stage of the research and development effort, measurements have been performed at a surface test facility on two $100 \mathrm{~mm}$ diameter, $33 \mathrm{~mm}$ thick cylindrical detector-grade germanium crystals, each of mass $1.4 \mathrm{~kg}$.

\section{Detector Design \& Housing}

These two germanium n-type crystals of orientation [001] were purchased from Umicore [8]. Each crystal has an impurity level of $\sim 10^{10}$ atoms $/ \mathrm{cm}^{3}$ and an etch-pitch density of $\sim 3000 \mathrm{~cm}^{-2}$. The crystals were etched, polished and cleaned in an ultrasonic bath prior to depositing the sensors on the flat surfaces of the crystals [9].

The tests were performed using existing CDMS-II cold hardware [10] with minor modifications. Each crystal is housed in a larger copper hexagonal prism of side length $61.5 \mathrm{~mm}$ and inscribed circle radius of $53 \mathrm{~mm}$. Wire bonds running from each channel connect to Device Interface Boards (DIBs) on faces of the hexagon for readout. 
The top lid has been designed to fasten to a conventional CDMS-II tower, and CDMS-II detector-to-tower wiring, termed vacuum side coaxes, connect each DIB to the tower. The $25 \mathrm{~mm}$ gap between the tower and coaxial wires is bridged by an extender plug. The remaining readout system is identical to the CDMS-II design.

The assembled tower is mounted within a cryostat and cooled to $50 \mathrm{mK}$ with an Oxford Instruments Kelvinox 100 dilution refrigerator [11]. The detector is controlled and readout with prototype SuperCDMS Detector Control and Readout Cards (DCRCs) [12] connected to a computer running a LabVIEW-based data acquisition software. Each DCRC is connected to a single DIB through the cold readout hardware and a timing link is established between cards to synchronize the digitizers.

The raw data files are transferred to a Linux cluster and processed with the standard CDMS analysis software package. Power Spectral Densities (PSDs) are built for each file from 500 noise traces acquired at the beginning of each data series. An optimal filter algorithm reconstructs the pulse amplitude and start time using a fit to an ideal pulse template in the frequency domain, while suppressing the noisy frequency bins. Poorly fitted pulses can be removed through a cut on the $\chi^{2}$ value from these fits.

\section{Charge Collection Efficiency Measurements}

Incident photons collide with target electrons in what is termed electron recoils. They can deposit sufficient energy to elevate these electrons across the bandgap from the valence to conduction band. This results in a cascade of electron-hole pairs. Applying an electric field across the crystal prevents recombination of these pairs. Instead, electrons drift toward the positive electrode. As electrons leave a normal valence site to fill an existing hole, thereby creating a new hole where they once were, the holes effectively move toward the opposite side of the crystal.

Unlike gamma-rays, incident neutrons and WIMPs interact with target nuclei in what is termed nuclear recoils. The ionization signal from nuclear recoils is quenched relative to that from electron recoils [13, 14]. Close to complete collection of these charge carriers is essential as the ionization signal is used to distinguish between recoil types, thus allowing for event-by-event discrimination [6]. One of the crystals is dedicated to assessing the charge collection efficiency of these larger germanium substrates. A detailed description of this detector's fabrication, testing and ionization results is given in [15]. These topics are briefly discussed here to introduce additional results.

Four electrodes are patterned as concentric rings on one face as shown in Fig. 1, and are wire bonded to the charge pins on the DIBs so that they can be read out and held at a potential. The electrodes are designated Q $n$, where the integer $n$ goes from 1 for the center electrode to 4 for that at the edge. For comparable responses, each of these electrodes cover the same surface area of the crystal. A single grounded electrode on the opposite side allows for an electric field to be applied across the crystal. Each electrode consists of a grid of $2 \mu \mathrm{m}$ thick traces at a pitch of $40 \mu \mathrm{m}$ and separated by $400 \mu \mathrm{m}$ wide trenches.

Four ${ }^{241} \mathrm{Am}$ sources are mounted in a holder sitting above the detector such that each lies facing the center of one of the electrodes. Collimation is provided by a hole of diameter $0.2 \mathrm{~mm}$ through a $1.6 \mathrm{~mm}$ thick lead disc, each source yielding an activity of $20 \mathrm{~Bq}$, mostly focused in the $60 \mathrm{keV}$ gamma line. 


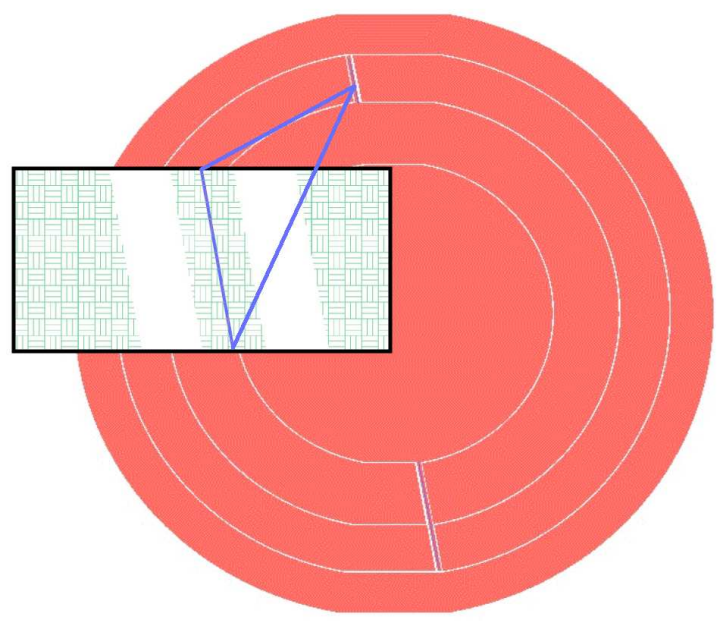

Figure 1: Mask used to pattern four ionization electrodes as a set of concentric rings (gray grid) on one side of a $100 \mathrm{~mm}$ diameter test crystal. The inset depicts a magnified view of the electrode grid pattern and demonstrates how the Q2 electrode extends through the white gaps that bisect the Q3 electrode, bringing it closer to the Device Interface Boards (DIBs) for wire bonding. In a similar fashion, the Q1 electrode extends through gaps that split the Q2 \& Q3 electrodes in the bottom-right of the figure. Additional wire bonds bridge these gaps, reconnecting the ends of each broken ring.

Through varying the bias applied to the electrodes, the electric field strength across the crystal can be controlled. Performing Gaussian fits to the $60 \mathrm{keV}$ peaks at various potentials leads to the distributions shown in Fig. 目 for the three inner charge channels. For these measurements, events where Q4 exhibits the dominant signal are vetoed to minimize crystal edge effects.

The charge collection efficiency is asymmetric because of the larger trapping and loss probability for electrons when compared to holes [16]. As the mean free path of $60 \mathrm{keV}$ photons in germanium is $\sim 1 \mathrm{~mm}$, the vast majority of events occur very close to the crystal face patterned with 4 electrodes. Therefore, for positive biases (positive electric field strengths in Fig. 2), electrons drift a short distance to the electrodes, while holes drift most of the distance across the crystal. The reverse is true for negative biases (negative electric field strengths in Fig. Z). Because of the relatively larger distance to traverse, when negative bias is applied to the electrodes the electrons have a higher chance of becoming trapped on their journey to the other face of the crystal.

Because of the relatively small mean free path of $60 \mathrm{keV}$ photons in germanium, additional measurements were performed to illuminate the bulk of the crystal by exposing the detector to a ${ }^{133} \mathrm{Ba}$ source external to the cryostat. The charge collection efficiencies from these events are calculated in the same way as those from the $60 \mathrm{keV}$ photons, and a comparison between bulk and surface electron recoils is shown in Fig. 2. The three inner electrodes exhibit similar responses and the charge collection efficiencies derived from both sources are consistent, indicating that there is little difference between events originating from the crystal bulk and those close to the surface.

It is preferable to apply as low an electric field as possible across the crystal to minimize the Luke phonon contribution [17]. A large contribution from Luke phonons results in noisier recoil energy and yield measurements, bringing the nuclear and electron recoil bands closer together thus 


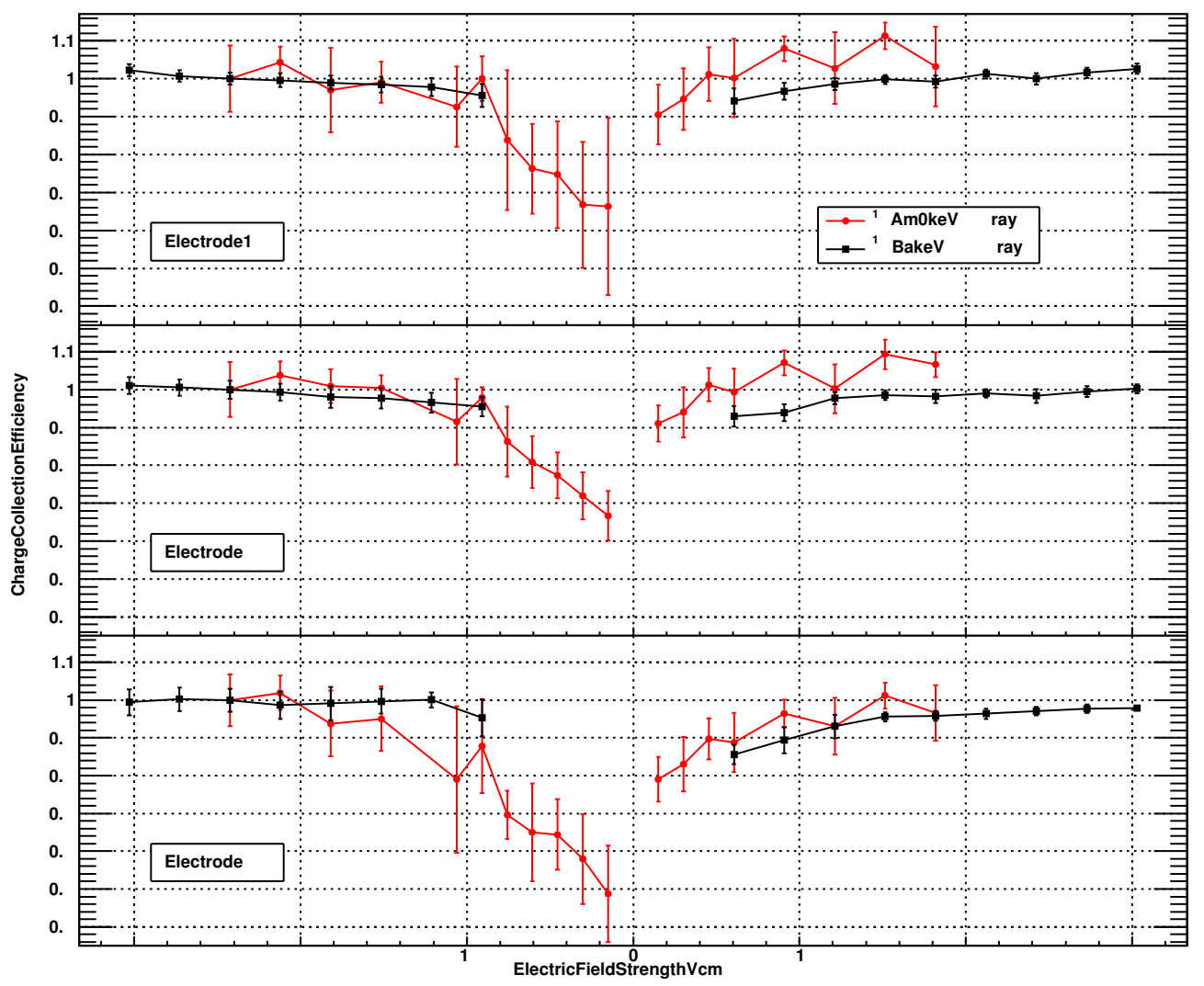

Figure 2: Charge collection efficiency as a function of electric field strength for Q1, Q2 \& Q3 electrodes from the $100 \mathrm{~mm}$ diameter ionization test crystal. Negative electric field strengths correspond to negative biases. Efficiencies are nomalized to unity at $-8 \mathrm{~V}$ (electric field strength of $-2.4 \mathrm{~V} / \mathrm{cm}$ ). Events where Q4 exhibits the dominant signal are vetoed. The charge collection efficiencies are consistent across all electrodes and there is a good agreement between $60 \mathrm{keV}$ events close to the crystal surface (red circles) and $356 \mathrm{keV}$ photons that penetrate the bulk (black squares).

reducing the discrimination power. Scaling past measurements [18] performed with $10 \mathrm{~mm}$ thick germanium crystals ( $30 \mathrm{~mm}$ diameter) indicates that an electric field of $0.5 \mathrm{~V} / \mathrm{cm}$ should be sufficient for efficient charge collection in $33 \mathrm{~mm}$ thick germanium detectors. To within measurement uncertainties, this agrees with the results in Fig. 2. This potential yields an electric field strength lower than that currently applied to $25 \mathrm{~mm}$ thick iZIP detectors in operation at the Soudan Underground Laboratory, implying that the larger germanium substrates have the necessary charge collection efficiency to be operated as dark matter detectors.

\section{Phonon Signals from the $100 \mathrm{~mm}$ Diameter iZIP}

An interaction in the crystal lattice that is induced by an incident particle results in the oscillation of atoms of that lattice. Phonons are the quantum mechanical description of these vibrations.

The second test crystal is convered into the first $100 \mathrm{~mm}$ diameter germanium iZIP. It features two electrodes on each crystal face, patterned as an inner disc and outer ring in a similar fashion to 


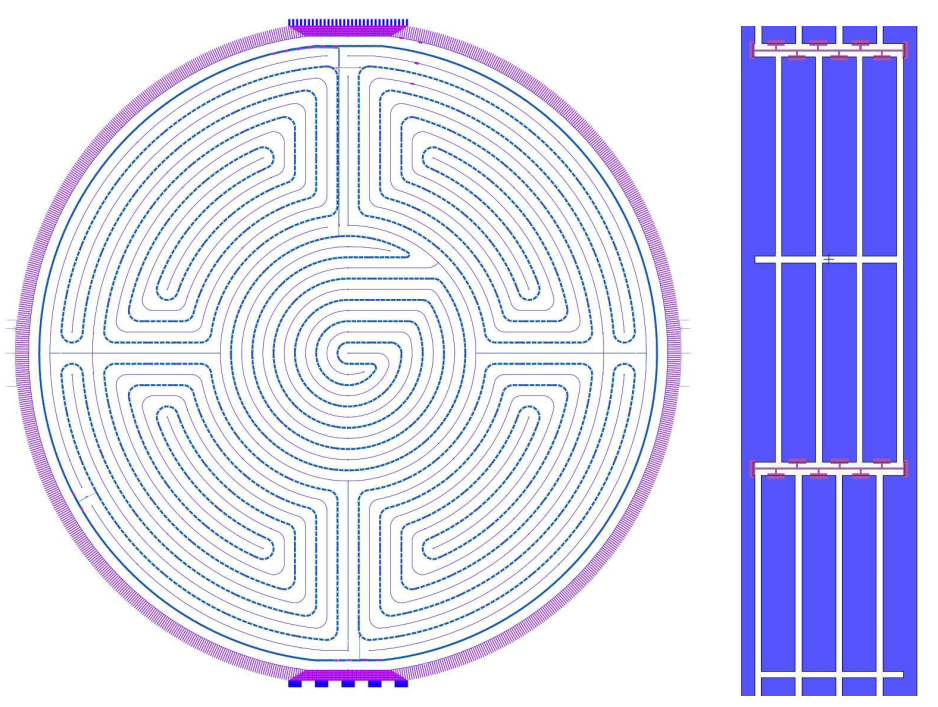

Figure 3: (Left) Mask design for surface of $100 \mathrm{~mm}$ diameter iZIP. Ionization electrodes are represented as thin purple strips that form two channels: one outer ring; and an inner disc. The electrodes are interleaved between phonon ribbons that are comprised of Quasiparticle trap assisted Electrothermal feedback Transition edge sensors (QETs). A total of six phonon channels are patterned on each side in the following configuration: one outer ring; one inner disc; and four of equal surface area covering the middle ring. (Right) Schematic of the QET, consisting of superconducting aluminum fins (blue) and tungsten traps and Transition Edge Sensors (purple) voltage biased in their superconducting transition region.

the $76 \mathrm{~mm}$ diameter iZIPs in operation at the Soudan Underground Laboratory. On each side, six phonon channels comprised of $260 \mu \mathrm{m}$ wide ribbons are arranged in three concentric rings of radii $19.2 \mathrm{~mm}, 44.8 \mathrm{~mm}$ and $50.0 \mathrm{~mm}$, shown in Fig. 3. The outer ring and inner disk consist of a single ribbon each. The middle ring consists of four phonon channels that cover an equal surface area. The $50 \mu \mathrm{m}$ wide ionization ribbons are interleaved with the phonon sensors at a pitch of $1.5 \mathrm{~mm}$. The same pattern for ionization and phonon sensors is repeated on the opposite crystal face and is rotated by $45^{\circ}$ about the central axis to improve event position information.

Each phonon channel is constructed from a collection of photolithographically patterned Quasiparticle trap assisted Electrothermal feedback Transition edge sensors (QETs) [19], shown in Fig. 3. They consist of $\sim 300 \mu \mathrm{m}$ long superconducting aluminum fins of width $50 \mu \mathrm{m}$, tungsten traps of width $27 \mu \mathrm{m}$ and overlap $4.5 \mu \mathrm{m}$ and tungsten Transition Edge Sensors (TESs) of length $215 \mu \mathrm{m}$. The tungsten is voltage biased such that it lies within its superconducting transition region. The critical temperatures of the tungsten TESs, defined as that where the transition region is reached when heating from the superconducting state, can be controlled during detector fabrication [9]. The critical temperatures of the phonon sensors are measured with a $\mathrm{RuO}_{2}$ thermometer thermally anchored to the tower and show relatively good uniformity, lying between $77 \mathrm{mK}$ and $85 \mathrm{mK}$.

When struck by phonons, Cooper pairs in the superconducting aluminum fins are broken. The unparied electrons, known as Bugoliubov quasiparticles, diffuse through to the tungsten traps. Upon entering the traps, the quasiparticles undergo electron-electron interactions raising the temperature of the adjacent TES. As the TES is voltage biased to remain in the transition region, a 

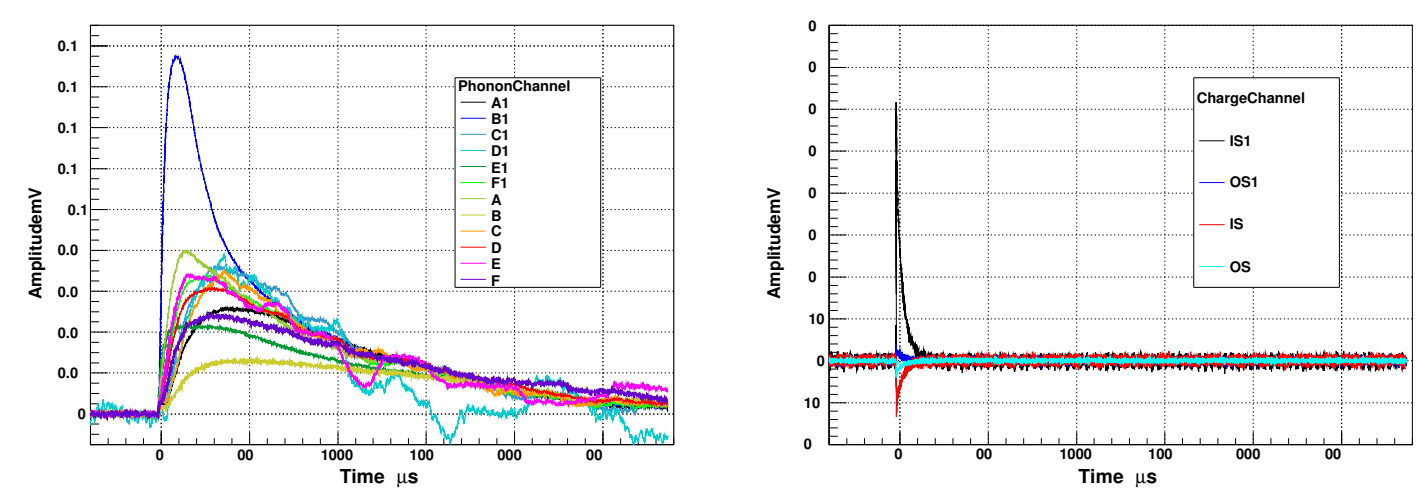

Figure 4: (Left) Phonon and (Right) ionization pulses from a $356 \mathrm{keV}$ electron recoil event. For the phonon channels, the letter and number correspond to the position and crystal face respectively. The steeper rise time and larger amplitude of phonon channel B1 indicates that this event lies close to this channel. Exploiting the isotropic phonon distribution after $\sim 1 \mathrm{~ms}$, a relative energy calibration is performed by normalizing the pulse tails. For the ionization channels, $Q I \& Q O$ represent the inner disc and outer ring respectively. The number designates the crystal face. The amplitude of the charge pulses is proportional to the deposited ionization energy and their decay time is fixed at $40 \mu$ s by the readout electronics [10].

small increase in temperature leads to a relatively large increase in resistance, which in turn lowers the current. The system returns to equilibrium between the superconducting and normal states as the Joule heating decreases with increasing resistance, lowering the temperature of the TES (this effect is know as electrothermal feedback). The current is monitored by a high-bandwidth SQUID array [10] and translated into pulses as shown in Fig. 4 .

The aluminum fins cover $5 \%$ of the crystal surface. As a result, not all phonons that reach the surface of the crystal are detected immediately. A significant fraction will undergo a number of reflections before coming into contact with a superconducting fin. Therefore, the first $\sim 1 \mathrm{~ms}$ of each phonon pulse contains position information, where relatively steeper rise times and larger amplitudes imply that the event in question occurs closer to this phonon sensor. After this time, the distribution of phonons is isotropic, and a relative energy calibration between channels can be performed by matching the pulse tails as shown in Fig. 7 . The phonon pulse decay times are measured to be $920 \pm 20 \mu \mathrm{s}$, in line with expectations.

\section{Surface Event Identification}

As discussed in Section 1, surface events can leak into the nuclear recoil band due to incomplete charge collection. The interleaved design permits identification of these surface events by applying a stronger electric field near the detector faces-between the biased ionization rails and the grounded phonon ribbons on the same side - than across the crystal bulk (between the biased ionization rails on opposite sides). Consequently, surface events result in side-asymmetric ionization signals whereas events in the bulk give rise to side-symmetric ionization signals, thus enabling surface event discrimination. 


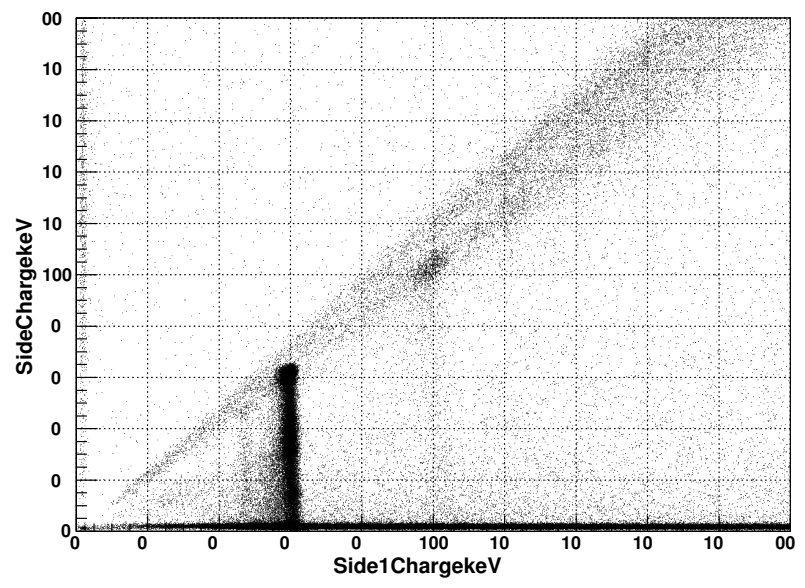

Figure 5: Comparison of ionization signals from $100 \mathrm{~mm}$ diameter iZIP sides $1 \& 2$. The large population of events that only register an ionization signal on side 1 correspond to $\beta$-particles emitted by the internal ${ }^{210} \mathrm{~Pb}$ source. The events along the diagonal are bulk events from Compton scatters of background gammarays. The vertical line represents $60 \mathrm{keV}$ photons from the internal ${ }^{241} \mathrm{Am}$ source that can have sufficient penetration depth in germanium to interact where the electric field across the bulk of the crystal dominates.

This principle has been demonstrated in the $76 \mathrm{~mm}$ diameter iZIP detectors in operation at the Soudan Underground Laboratory [6]. To test that the larger germanium substrate iZIP detector described in this article also allows for the identification of surface events, the crystal was exposed to internal ${ }^{210} \mathrm{~Pb}$ and ${ }^{241} \mathrm{Am}$ sources. Both sources faced crystal side 1 . A comparison of the ionization signals from crystal sides $1 \& 2$ is shown in Fig. 5. A clear population of surface events originating from the $\beta$-particles emitted by the ${ }^{210} \mathrm{~Pb}$ source yields no ionization signal on side 2 . These electrons have a relatively short mean free path length in germanium of $\sim 1 \mu \mathrm{m}$. Additionally, the $60 \mathrm{keV}$ gamma-rays emitted from ${ }^{241} \mathrm{Am}$ yields a vertical line as some photons reach a sufficient penetration depth to interact in the bulk of the crystal.

Therefore, surface events can be identified in a similar fashion to the current generation of $76 \mathrm{~mm}$ diameter detectors. Although not discussed here, additional methods to identify surface events used in CDMS-II based on phonon side asymmetry and pulse shape [2] can also be applied. A study to quantify the surface event rejection efficiency for these larger germanium substrates is currently in progress.

\section{Conclusions}

The $100 \mathrm{~mm}$ diameter germanium crystals have the necessary charge collection efficiency to be operated as next generation dark matter detectors. Detection principles relative to the current generation of $76 \mathrm{~mm}$ diameter iZIP detectors, such as surface event rejection, can be applied to the larger germanium substrates tested here. The next steps include the determination of the surface event rejection efficiency and demonstration of electron-nuclear recoil discrimination. 


\section{Acknowledgments}

This work is supported in part by the National Science Foundation (Grant Nos. PHY-0705052, PHY-0902182 \& PHY-1004714) and the Department of Energy (Contracts DE-AC02-07CH00359, DE-AC02-76SF00515 \& DOE-ER-40823-2500).

\section{References}

[1] G. Jungman, M. Kamionkowski \& K. Griest, Supersymmetric dark matter, Phys. Rep. 267 (1996) 195.

[2] Z. Ahmed et al., Dark matter search results from the CDMS-II experiment, Science 327 (2010) 1619 [0912.3592 [astro-ph.co]].

[3] Z. Ahmed et al., Results from a low energy analysis of the CDMS II germanium data, Phys. Rev. Lett. 106 (2011) 131302 [1011.2482 [astro-ph. Co]].

[4] P. N. Luke et al., Calometric ionization detector, Nucl. Instrum. Methods Phys. Res. A289 (1990) 406.

[5] P. L. Brink et al., First test runs of a dark matter detector with interleaved ionization electrodes and phonon sensors for surface-event rejection, Nucl. Instrum. Methods Phys. Res. A559 (2006) 414.

[6] R. Agnese et al., Demonstration of surface electron rejection with interleaved germanium detectors for dark matter searches, Appl. Phys. Lett. 103 (2013) 164105 [1305. 2405 [physics.ins-det]].

[7] P. L. Brink, Conceptual design for SuperCDMS SNOLAB, J. Low Temp. Phys. 167 (2012) 1093.

[8] Umicore Electro-Optic Materials, Waterforenstraat 33, 2250 Olen, Belgium.

[9] P. L. Brink et al., SuperCDMS Detector Fabrication Advances, AIP Conf. Proc. 1185 (2009) 655.

[10] D. S. Akerib et al., Design and performance of a modular low-radioactivity readout system for cryogenic detectors in the CDMS experiment, Nucl. Instrum. Methods Phys. Res. A591 (2008) 476.

[11] Oxford Instruments, Tubney Woods, Abingdon, Oxfordshire OX13 5QX, United Kingdom.

[12] S. Hansen et al., The Cryogenic Dark Matter Seach test stand warm electronics card, in Nuclear Science Symposium Conference Record (NSS/MIC), 2010 IEEE (2010) 1329.

[13] J. Lindhard, M. Scharff \& H. E. Schiøtt, Range concepts and heavy ion ranges (notes on atomic collisions, II), Mat. Fys. Medd. Dan. Vid. Selsk. 33 (1963) 14.

[14] J. Lindhard et al., Integral equations governing radiation effects (notes on atomic collisions, III), Mat. Fys. Medd. Dan. Vid. Selsk. 33 (1963) 10.

[15] H. Chagani et al., Ionization measurements of SuperCDMS SNOLAB $100 \mathrm{~mm}$ diameter germanium crystals, J. Low Temp. Phys. 167 (2012) 1125.

[16] K. M. Sundqvist et al., Transport and two-species capture of electrons and holes in ultrapure germanium at milliKelvin temperature, J. Low. Temp. Phys. 176 (2014) 188.

[17] P. N. Luke, Voltage-assisted calorimetric ionization detector, J. Appl. Phys. 64 (1988) 6858.

[18] T. A. Shutt, A Dark Matter detector based on the simultaneous measurements of phonons and ionization at $20 \mathrm{mK}$, Ph.D. Thesis, Department of Physics, University of California, Berkeley (1993).

[19] K. D. Irwin \& G. C. Hilton, Transition-Edge Sensors in Cryogenic Particle Detection, Topics Appl. Phys. 99 (2005) 63. 